\title{
SALIVARY ANTISENSE NON-CODING RNA IN THE INK4 LOCUS \& MATERNAL EXPRESSED GENE 3 AS EARLY BIOMARKERS IN DETECTION OF MALIGNANT TRANSFORMATION OF ORAL LICHEN PLANUS
}

\author{
Dalia M. Ghalwash*, Olfat G. Shaker**, Yasmine Elshiwy*** and Noha A. Radwan ${ }^{* * * *}$
}

\begin{abstract}
Oral lichen planus (OLP) is a potentially malignant lesion. It is crucial to properly identify patients at risk of malignant transformation. LncRNA antisense non-coding RNA in the INK4 locus (ANRIL) has been known to advance tumor progression. Another type of lncRNAs is maternal expressed gene (MEG3) which is a tumor suppressor gene that can inhibit the advancement of malignancy. The goal of this study was to outline the relative fold expression of ANRIL and MEG3 in OLP and oral squamous cell carcinoma (OSCC). The study included 20 histopathologically diagnosed OLP patients and 10 patients with OSCC. The relative fold expression of ANRIL and MEG3 in the OLP group was compared to OSCC group using qRT-PCR. The results showed that the relative fold expression of ANRIL was significantly increase in the OSCC compared to OLP group $(\mathrm{p}<0.01)$. ROC analysis showed that with cut-off value of 4.1 relative fold expression of ANRIL with sensitivity 0.8 , specificity 0.7 and AUC 0.85 . The decrease in MEG3 was insignificant. ANRIL relative fold expression could perform an essential part in OSCC progression, and could assist in the future as a potential biomarker for malignant transformation of OLP.
\end{abstract}

KEYWORDS: LncRNAs - oral squamous cell carcinoma - qRT-PCR - salivary sample ROC curve.

\section{INTRODUCTION}

Oral lichen planus (OLP) is a chronic mucocutaneous disease. It affects around $1 \%-2 \%$ of the population ${ }^{[1]}$. Patients with OLP should be closely monitored as the World Health Organization (WHO) has referred to OLP as a "potentially malignant disorder." The malignant transformation rate documented around $1 \%{ }^{[2]}$.

\footnotetext{
* Associate Professor of Oral Medicine and Periodontology, Faculty of Dentistry, The British University in Egypt (BUE), Cairo, Egypt.

** Professor of Biochemistry, Faculty of Medicine, Cairo University, Cairo, Egypt.

***Associate Professor of Clinical and Chemical Pathology, Faculty of Medicine, Cairo University, Cairo, Egypt.

****2ecturer of Clinical and Chemical Pathology, Faculty of Medicine, Cairo University, Cairo, Egypt.
} 
Oral squamous cell carcinoma (OSCC) is considered one of the most aggressive diseases accountable for more than $90 \%$ of all oral cancers ${ }^{[3]}$. The leading reasons of OSCC-induced mortality are metastasis and recurrence ${ }^{[4]}$. The total survival rate in the majority of countries ranges around $45 \%$, despite vast evolutions in the field of diagnostics and therapeutics ${ }^{[5]}$. To identify tumor-specific biomarkers useful in early diagnosis and management of the disease, further clarification is needed for the molecular mechanisms triggering tumorigenesis in $\mathrm{OSCC}^{[4]}$.

Long non-coding RNAs (LncRNAs) are an innovative category of mRNA-like transcripts. LncRNAs accelerate cancer cells proliferation and metastasis, and impact cancer development and progression $^{[4]}$. In a large range of malignancies as breast cancer, LncRNAs have been found to be dysregulated ${ }^{[6]}$.

Antisense Non-coding RNA in the INK4A Locus (ANRIL), located in the 9p21 region, is a type of LncRNA that was recognized as a hotspot in several cancers ${ }^{[7]}$. ANRIL suppression in OSCC cells drastically repressed cell proliferation and invasion. These findings recommend ANRIL to play a crucial part as a diagnostic and therapeutic target in $\mathrm{OSCC}^{[4]}$.

MEG3, located in the $14 \mathrm{q} 32.3$, is lncRNA encoded by a maternally imprinted gene (MEG3) associated with tumorigenesis. In a variety of human tumor cells, the expression of the MEG3 was downregulated ${ }^{[8]}$.

In the current study, we characterized the level of ANRIL and MEG3 level in the OLP lesions, either erosive or atrophic, and OSCC lesions to investigate the possibility of using these LncRNAs as an early marker for progression of OLP lesions into OSCC. To the best of our knowledge regarding the role of LncRNAs in OLP lesions, this is the first study to impart innovative understandings about this topic.

\section{MATERIALS AND METHODS}

\section{Subjects}

In the current study, 20 OLP patients (10 with erosive OLP and 10 with atrophic OLP), 10 OSCC patients, confirmed by pathological and clinical diagnoses, and 20 normal control subjects at the Faculty of Dentistry Cairo University were enrolled between December 2017 and August 2018. This study was approved by the Ethical Committee of the Faculty of Dentistry Cairo University. Consent was taken from all patients. A thorough intraoral examination were carried out for all patients by the oral medicine specialist (DG) and patients with clinical or histopathological confirmation for atrophic and erosive lichen planus were included in the study. Lesions were mostly located in the buccal mucosa and on the tongue.
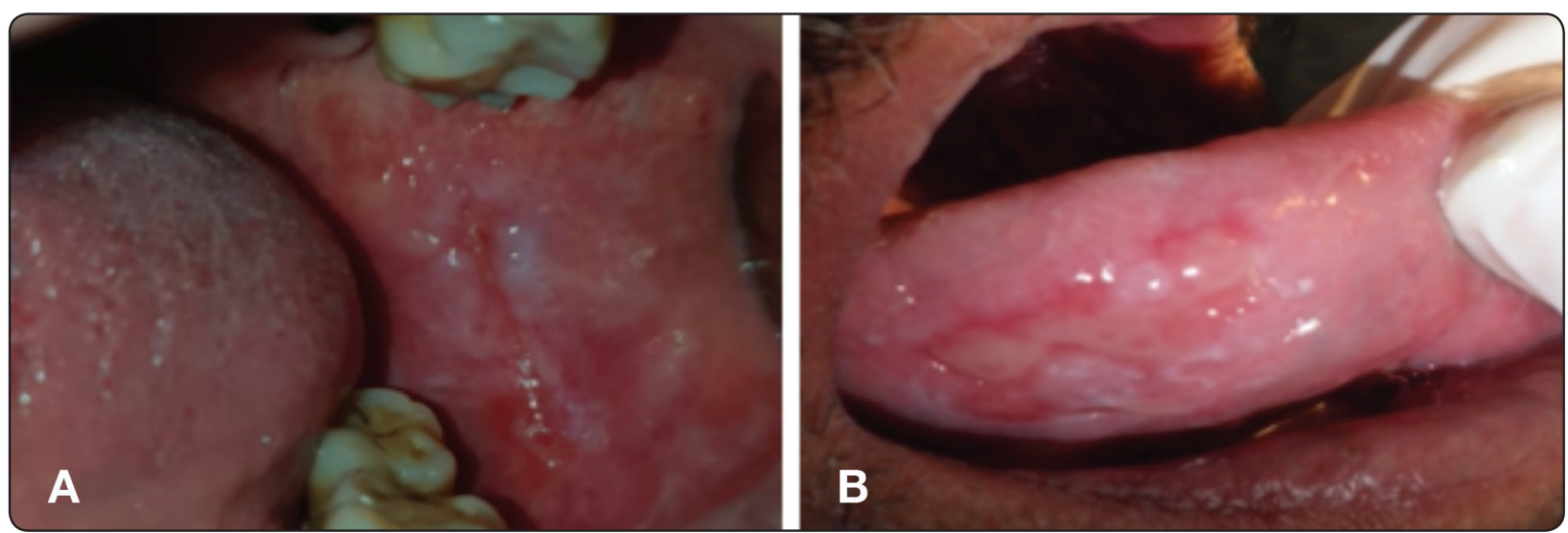

Fig. (1): A. 49-year-old female patient with atrophic OLP on buccal mucosa. B. 45-year-old male patient with bollus erosive OLP on the tongue. 


\section{RNA extraction and qRT-PCR}

The saliva samples were collected in sterile eppendorfs $(0.5 \mathrm{~mL})$. MiRNeasy extraction kit (Qiagen, Valenica, CA) was utilized for extraction of total RNA from saliva by using QIAzol lysis reagent following the manufacturer's guidelines. A final volume of $20 \mu \mathrm{l}$ RT reactions containing $60 \mathrm{ng}$ of total RNA utilizing RT2 first strand Kit (Qiagen, Valenica, CA) following the manufacturer's instructions. Using GAPDH as internal control, Expression levels of the lncRNAs (ANRIL and MEG3) were calculated in saliva using readymade primers and Maxima SYBR Green PCR kit (Thermo, USA) following the manufacturer's protocol. Briefly, $20 \mu \mathrm{l}$ reaction mixture was prepared by mixing $10 \mu \mathrm{l}$ master mix, $1 \mu \mathrm{l}$ primer and cDNA. Real-time PCR was carried out on the prepared mixture by using Rotor gene Q System (Qiagen) with the ensuing conditions: $95{ }^{\circ} \mathrm{C}$ for $10 \mathrm{~min}$, followed by 45 cycles at $95{ }^{\circ} \mathrm{C}$ for $15 \mathrm{~s}$ and $60{ }^{\circ} \mathrm{C}$ for $60 \mathrm{~s}$. The cycle threshold $(\mathrm{Ct})$ is the number of cycles essential for the fluorescent signal to cross the threshold in real-time PCR. Relative to the internal control, calculation of gene expression was done by $2^{-\Delta \mathrm{Ct}}$. Calculating the relative fold expression was done using $2^{-\Delta \Delta \mathrm{Ct}[9]}$.

\section{Statistical analysis}

Statistical analysis was performed using SPSS 18.0 software (IBM, SPSS, Chicago, IL, USA). A D'Agostino-Pearson Test was used to check for normal distribution of data. ANOVA test with Bonferroni corrected $p$-value was used to compare between more than two groups, while, Student's t-test was used to compare between each two groups separately and the data are shown as mean \pm standard deviation (SD) for normally distributed parameters. The receiver-operating characteristics (ROC) curve was depicted to establish the predictive value of the ANRIL and MEG3 variants in the detection of the malignant transformation from OLP to OSCC. Also, the slope of the ROC curve indicated the likelihood ratio (LR) for a diagnostic test.

\section{RESULTS}

Up-regulation of Expression of ANRIL in OLP and $\mathrm{OSCC}$

In this study, we analysed the increase in relative fold change of expression of ANRIL (Fig. 1) between OLP patients and OSCC patients (Table 1) which revealed significantly up-regulation in relative fold change in OLP group (either atrophic or erosive) than OSCC (Table 1).

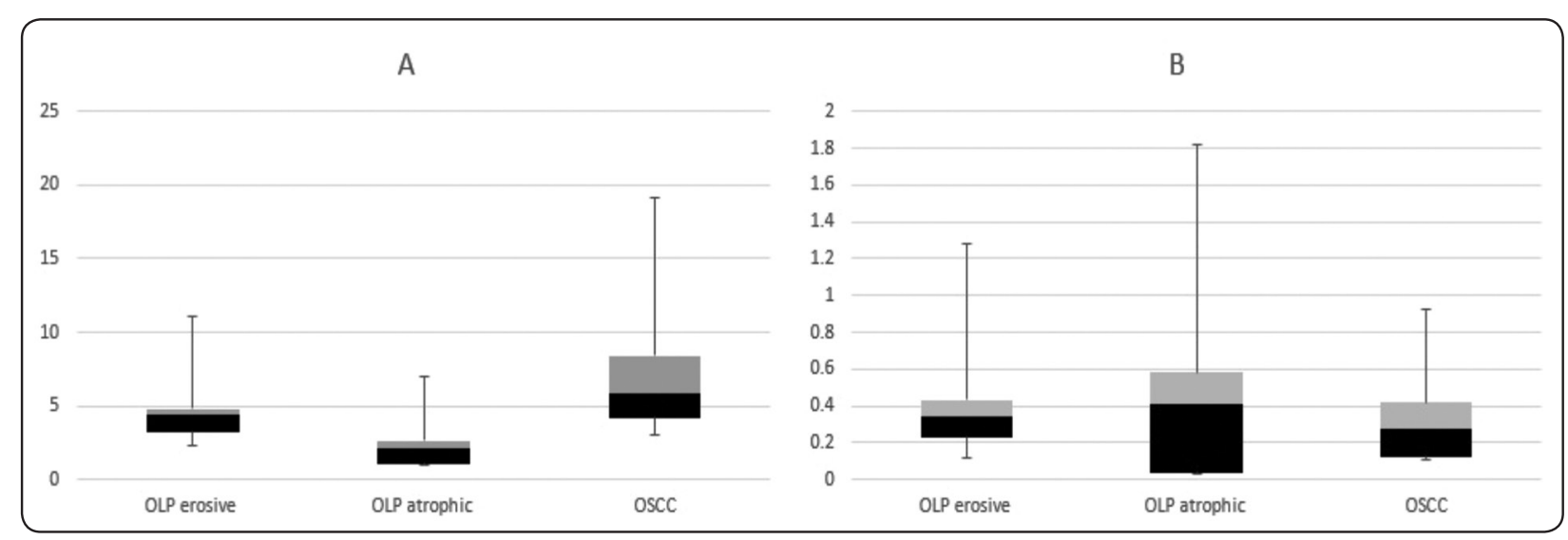

Fig. (2): This box and whisker plot shows: a) The relative fold change of expression of ANRIL OLP erosive, OLP atrophic and OSCC groups. b) The relative fold change of expression of MEG3 in OLP erosive, OLP atrophic and OSCC groups. 
TABLE (1): The relative fold expression of ANRIL and MEG3 in OLP and OSCC.

\begin{tabular}{|c|c|c|c|}
\hline & & Mean & \\
\hline \multirow{3}{*}{$\begin{array}{c}\text { ANRIL relative fold } \\
\text { expression (Up-regulation) }\end{array}$} & OLP atrophic $\left(n^{* *}=10\right)$ & $2.2,95 \%$ CI $[1.456,2.944]$ & \multirow{3}{*}{$p<0.00^{*}$} \\
\hline & OLP erosive $(n=10)$ & $4.2,95 \%$ CI $[3.456,4.944]$ & \\
\hline & $\operatorname{OSCC}(n=10)$ & $6.3,95 \%$ CI $[4.627,7.973]$ & \\
\hline \multirow{3}{*}{$\begin{array}{l}\text { MEG3 relative fold expression } \\
\text { (Down-regulation) }\end{array}$} & OLP atrophic $(\mathrm{n}=10)$ & $0.4,95 \%$ CI $[0.152,0.648]$ & \multirow{3}{*}{$p=0.47$} \\
\hline & OLP erosive $(n=10)$ & $0.4,95 \%$ CI $[0.276,0.524]$ & \\
\hline & $\operatorname{OSCC}(n=10)$ & $0.3,95 \%$ CI $[0.176,0.424]$ & \\
\hline \multirow{3}{*}{$\begin{array}{c}\text { ANRIL relative fold } \\
\text { expression } \\
\text { (Up-regulation) }\end{array}$} & \multirow{2}{*}{ OSCC group } & OLP (Atrophic group) & $p<0.00 * * *$ \\
\hline & & OLP (Erosive group) & $p<0.03 * * *$ \\
\hline & OLP (Atrophic group) & OLP (Erosive group) & $p<0.00 * * *$ \\
\hline \multirow{3}{*}{$\begin{array}{l}\text { MEG3 relative fold expression } \\
\text { (Down-regulation) }\end{array}$} & \multirow{2}{*}{ OSCC group } & OLP (Atrophic group) & $p=0.3 * * *$ \\
\hline & & OLP (Erosive group) & $p=0.3^{* * *}$ \\
\hline & OLP (Atrophic group) & OLP (Erosive group) & $p=0.6^{* * *}$ \\
\hline
\end{tabular}

*ANOVA test with Bonferroni corrected p-value

\section{Expression of MEG3 in OLP and OSCC}

In this study, we investigated the down-regulation in the relative fold expression of MEG3 (Fig. 1) between OLP atrophic, OLP erosive and OSCC groups (Table 1) which was insignificantly downregulated between OLP group (either atrophic or erosive groups) and OSCC group (Table 1).

\section{ROC curve analysis for ANRIL and MEG3}

By plotting the sensitivity and 1- specificity on the $\mathrm{y}$-axis and the $\mathrm{x}$-axis respectively, the ROC curve is created. As the ROC curve rises briskly towards the left upper corner of the curve, it denotes good distinction function. According to the best equilibrium between sensitivity and specificity, the optimal cut-off points were chosen.

Level of ANRIL fold expression which provided the best discrimination between OLP and OSCC was equal to 4.1 fold expression. This cut-off point provided $80 \%$ sensitivity, $70 \%$ specificity and 0.85 AUC. The LR's of 2.7 denote that ANRIL levels more than or equal to the cut-off points were more likely to be found in OSCC than in OLP by 2.7 times $(p<0.00)$. As for MEG3 fold expression the AUC which was 0.4 indicated that MEG3 cannot be as a diagnostic biomarker for malignant transformation in OLP lesions.

\section{DISCUSSION}

OLP is a chronic disease of unknown aetiology with substantial influence on patients' quality of life. Malignant transformation into OSCC is considered as one of the most serious complications of the disease ${ }^{[1]}$. A malignant transformation rate of $1.4 \%$ was described by Bandyopadhyay et al. which was in concordance with the studies of Fang et al., Shen et al., Chainani-Wu et al., Eisen, Gorsky et al., Rajentheran et al., Markopoulos et al., and Kesic et al. While, Lo Muzio et al., Lanfranchi-Tizeira et $a l$., and Kaplan et al. reported a higher malignant transformation rate of $4.9 \%, 6.5 \%$, and in Italian and Argentinian population, respectively ${ }^{[2]}$.

The prognosis of patients with OSCC remains inadequate in spite of great evolution in early 
diagnosis and chemotherapy. Consequently, an advanced insight of the molecular mechanisms regarding OSCC is crucial to enhance the clinical choices, strategies and outcomes of patients ${ }^{[4]}$. The detection of lncRNAs has significantly changed our understanding of the mechanisms of progression of malignancies and surfaced as a novel and fundamental level of gene regulators ${ }^{[10]}$. Liu et al. found that the expression of ANRIL was greater in OSCC tissues compared to nearby non-tumor tissues ${ }^{[4]}$. The significant reduction of expression of MEG3 in OSCC was reported by Liu et al. Inhibition of the proliferation of cancer cells caused by MEG3 overexpression which also promoted apoptosis ${ }^{[11]}$. In the present study, the expression of ANRIL and MEG3 were reported in the Egyptian patients in comparison between OLP and OSCC for the first time.

In the current study, we found that ANRIL expression was significantly up-regulated in the OSCC than the OLP group (either the atrophic group or the erosive group). This rise in ANRIL expression highlights the clinical significance and a possibly essential role in predicting the advancement of OLP lesions into OSCC. On the other hand, we found that the down-regulation of MEG3, as a tumor suppressor gene, in the OLP groups (either the atrophic group or the erosive group) and OSCC group, was insignificant.

The search for new non-invasive circulating biomarkers was very important in early diagnosis of malignant transformation of OLP lesions and affecting treatment surveillance. We proposed that when ROC curve analysis was applied, the cut-off value of 4.1 relative fold expression (sensitivity 0.8 and specificity 0.7 ) could be used as an indicator to malignant transformation in OLP lesions. While using ROC curve in MEG3 relative fold expression revealed AUC 0.4 which indicated that MEG3 could not be used as an indicator in malignant transformation in OLP.
To our knowledge, our study was the first report in the salivary ANRIL and MEG3 relative fold expression in malignant transformation of OLP. ANRIL acted as valuable non-invasive biomarker in malignant transformation in OLP. However, there were still some limitations of this study. The first was the small sample size of OLP (20 cases) and OSCC patients (10 cases) and controls and we could not make further analysis such as the role of ANRIL in different subtypes and early stages of OSCC. The second limitation of our study was the lack of analysis between ANRIL levels and poor prognosis, the reason was that the enrolled patients were already diagnosed no prognosis information were available. Further studies should focus on the role of ANRIL in prediction of poor prognosis and with larger sample size.

This current study can pave the way to include the molecular prognostic markers LncRNAs e.g. ANRIL and MEG3 as early biomarkers to predict the prognosis of OLP lesion and the early detection of the malignant transformation of OLP into OSCC.

\section{Compliance with Ethical Standards}

Conflict of interest: All authors declare no conflict of interest.

Funding: No Funding.

Ethical approval: This article does not contain any studies with human participants or animals performed by any of the authors.

Informed consent: Informed consent was obtained from all individual participants included in the study.

\section{REFERENCES}

1. Tampa M, Caruntu C, Mitran M, Mitran C, Sarbu I, Rusu L, Matei C, Constantin C, Neagu M, Georgescu SR(2018). Markers of Oral Lichen Planus Malignant Transformation. Dis markers. DOI: 10.1155/2018/1959506.

2. Bandyopadhyay A, Behura SS, Nishat R, Dash KC, Bhuyan L, Ramachandra S (2017). Clinicopathological 
profile and malignant transformation in oral lichen planus: A retrospective study. J Int Soc Prevent Communit Dent. 7 (3): 116-24.

3. Silveira W, Bottezini E, Linden M, Rinaldi I, Paranhos L, Carli J, Trentin M,Letícia dos Santos P (2017). Squamous cell carcinoma from oral lichen planus: a case report of a lesion with 28 years of evolution. J Korean Assoc Oral Maxillofac Surg. 43 (1): S14-18.

4. Liu F, Liu S, Chen L, Lei Z, Wu J, Wu J (2017). Upregulation of the long non-coding RNA ANRIL indicates poor prognosis and promotes tumorigenesis in oral squamous cell carcinoma. Int J Clin Exp Pathol.10 (6): 6900-6905.

5. Majumdar B, Patil S, Sarode S, Sarode G, Rao SR (2017). Clinico-pathological prognosticators in oral squamous cell carcinoma: An update. Transl Res in Oral Oncol. 2: 1-14.

6. Tang H, Wu Z, Zhang J, SU B (2013). Salivary lncRNA as a potential marker for oral squamous cell carcinoma diagnosis. Mol Med Rep. 7 (3): 761-766.

7. Sarkar D, Oghabian A, Bodiyabadu PK, Joseph WR,
Leung EY, Finlay GJ (2017). Multiple Isoforms of ANRIL in Melanoma Cells: Structural Complexity Suggests Variations in Processing. Int J Mol Sci. 18 (7): 1378. Doi:10.3390/ijms18071378.

8. Murray R, Bryant J, Titcombe P, Barton SJ, Inskip H Harvey NC (2016). DNA methylation at birth within the promoter of ANRIL predicts markers of cardiovascular risk at 9 years. Clin Epigenet. 8: 90.

9. Livak KJ, Schmittgen TD (2001). Analysis of relative gene expression data using real-time quantitative PCR and the 2 (-Delta Delta C (T)) Method. Methods. 25 (4): 402-8.

10. Huang M, Chen W, Qi F, Xia R, Sun M, Xu T, Yin L, Zhang E, De W, Shu Y (2015). Long non-coding RNA ANRIL is upregulated in hepatocellular carcinoma and regulates cell apoptosis by epigenetic silencing of KLF2. J Hematol Oncol. 8:50.

11. Liu Z, Wu C, Xie N, Wang P (2017). Long non-coding RNA MEG3 inhibits the proliferation and metastasis of oral squamous cell carcinoma by regulating the $\mathrm{WNT} / \beta$ catenin signaling pathway. Oncol Lett. 14 (4): 4053-4058. 